Int. J. Morphol.,

28(1):249-253, 2010

\title{
Densidad Neuronal en la Corteza Visual Primaria (Área 17), en Dos Especies de Octodon
}

\author{
Neuronal Density in Primary Visual Cortex (17 Visual Area), in Two Species of Octodon
}

"Olivares, R.; **Ortíz, A.; **Henríquez, M.; **Adaro, L. \& ***Aboitiz, F.

OLIVARES, R.; ORTÍZ, A.; HENRÍQUEZ, M.; ADARO, L. \& ABOITIZ, F. Densidad neuronal en la corteza visual primaria (área 17), en dos especies de octodon. Int. J. Morphol., 28(1):249-253, 2010.

RESUMEN: Estudios experimentales demuestran que modificaciones medioambientales pueden producir alteraciones en el desarrollo normal de la corteza cerebral visual y sus conexiones. Por otra parte, es posible que en condiciones naturales, las especies animales hayan desarrollado adaptaciones genéticas a las distintas condiciones de luminosidad en que realizan su actividad. Recientemente, se han observado variaciones significativas en la densidad neuronal cortical del área 17 (área visual primaria), en roedores silvestres con diferentes períodos diarios de actividad y relación filogenética distante (Abrothrix olivaceus y Phyllotis darwini), pero aún no se ha determinado la naturaleza genética o plástica de dichas diferencias. En este trabajo se compararon especies con una mayor cercanía filogenética, para disminuir al máximo la variable taxonómica. Se estudió la corteza visual primaria (área 17), de roedores silvestres nativos, de las especies Octodon degus $(\mathrm{n}=5)$ y Octodon bridgesi $(\mathrm{n}=3)$, pertenecientes a la Familia Octodontidae, con el propósito de evidenciar cambios a través de la medición de la densidad neuronal, mediante la técnica del disector óptico, en cortes de 40 $\mu \mathrm{m}$, incluidos en celoidina y teñidos con Nissl. Complementariamente, se realizó una cuantificación de la densidad neuronal de la corteza motora de las especies en estudio. $O$. degus, que presenta un período de actividad diurna, evidenció una densidad neuronal menor en la corteza visual $\left(34,32 \pm 2,51 \times 10^{4}\right.$ neuronas $\left./ \mathrm{mm}^{3}\right)$, que la observada en $O$. bridgesi $\left(39,55 \pm 0,64 \times 10^{4}\right.$ neuronas $\left./ \mathrm{mm}^{3}\right)$, especie de período de actividad nocturna; lo cual fue estadísticamente significativo $(\mathrm{t}=3,44 ; \mathrm{p}<0,05)$. Las diferencias encontradas se podrían relacionar con el tipo de condiciones de luminosidad en que se desenvuelven dichas especies, aunque no se puede descartar la influencia de otros factores.

PALABRAS CLAVE: Corteza cerebral; Corteza visual; Densidad neuronal; Octodon degus; Octodon bridgesi.

\section{INTRODUCCIÓN}

Evidencias experimentales en roedores, demuestran, que la organización final del sistema nervioso depende de factores no genéticos que actúan en el período prenatal y postnatal temprano. Entre ellos, la desnutrición que puede provocar efectos dañinos en el desarrollo del cerebro si se produce en el período de mayor susceptibilidad del mismo (Soto-Moyano et al., 1999).

Después del nacimiento, las exposiciones a los estímulos visuales modulan el desarrollo cortical, induciendo numerosos cambios en todos los componentes de la corteza visual. Es así como, se ha evidenciado que ratas criadas en oscuridad, versus animales criados con ciclos de luz - oscu- ridad, presentan menor densidad vascular en la capa IV de la corteza visual (Argandoña \& Lafuente, 2000).

Sin embargo, no queda claro si estas diferencias se deben a factores de plasticidad o de adaptación genética. Recientemente, se han observado variaciones en la densidad neuronal de la corteza visual primaria (área 17) en roedores silvestres, con diferentes períodos de actividad diaria. Abrothrix olivaceus, que presenta un período de actividad continuo a lo largo del día en la zona central de Chile, evidenció una densidad neuronal menor, estadísticamente significativa, que la observada en Phyllotis darwini, especie de actividad nocturna en la misma región del país (Olivares et

\footnotetext{
* Departamento Ciencias Biológicas Animales, Facultad Ciencias Veterinarias y Pecuarias, Universidad de Chile.

** Escuela de Medicina Veterinaria, Facultad de Ecología y Recursos Naturales, Universidad Andrés Bello.

**** Laboratorio de Neurobiología, Centro de Investigaciones Médicas, Pontificia Universidad Católica de Chile.

Financiamiento: Fondo de Investigación Veterinaria (FIV) N 9102081
} 
al., 2004). Por lo anterior, se puede suponer que en forma natural, diversos grados de luminosidad (diurna, crepuscular o nocturna), podrían modular de diversas formas, el desarrollo de la corteza visual.

Es así como, al comparar especies más cercanas filogenéticamente, de manera de minimizar la variable taxonómica (Felsenstein, 1985), podríamos pensar que las diferencias observadas pudieran deberse a plasticidad.

Se ha observado que la familia Octodontidae (Rodentia), incluye especies nocturnas y crepusculares, particularmente entre especies del género Octodon (Honeycutt et al., 2003). Un modelo de actividad diurna-crepuscular ha sido descrito en $O$. degus tanto en estado silvestre (Kenagy et al., 2002), como en cautiverio (Hernández et al., 2003). Por otro lado, $O$. bridgesi presenta una actividad nocturna en capturas a terreno (Verzi \& Alcover, 1990).

El objetivo de este trabajo fue estudiar la corteza visual primaria (área 17), de dos especies de roedores silvestres nativos, Octodon degus (degú de las pircas) y Octodon bridgesi (degú de Bridges), con el propósito de evidenciar eventuales variaciones en la densidad neuronal cortical occipital, que pueden explicar la distinta actividad conductual.

\section{MATERIAL Y MÉTODO}

Se obtuvieron encéfalos $O$. degus (n=5, peso corporal promedio $170,6 \pm 69,7 \mathrm{~g}$, peso encefálico promedio $2,7 \pm 0,2 \mathrm{~g})$ у $O$. bridgesi $(\mathrm{n}=3$, peso corporal promedio $94,3 \pm 9,2 \mathrm{~g}$, peso encefálico promedio $2,8 \pm 0,1 \mathrm{~g}$ ), provenientes de ejemplares capturados a campo, con los permisos correspondientes (SAG y CONAF). Los animales fueron sacrificados a través de una cámara de $\mathrm{CO}_{2}$ al $100 \%$, procedimiento aprobado por el Comité de Bioética Animal de la Facultad de Ciencias Veterinarias y Pecuarias de la Universidad de Chile.

Estos fueron fijados y conservados en formolaldehído tamponado al 10\%. En una segunda etapa, dichos encéfalos fueron lavados en agua abundante por un período de 24 horas para luego ser incluidos en celoidina de acuerdo a la técnica descrita por Yakovlev (1970).

Una vez impregnados los encéfalos en celoidina y endurecido el material, se realizaron cortes coronales anteroposteriores, de $40 \mu \mathrm{m}$ de grosor, los que fueron teñidos con Cresyl Violeta (Nissl) al 1\%, a fin de poder visualizar con mayor facilidad los somas neuronianos (Soto-Moyano et al.).
Se seleccionaron los cortes correspondientes al área dorsomedial del área 17 y, dentro de éstos, las capas IV y V, tomando como referencia el mapa estereotáxico de la citoarquitectura de la corteza cerebral presente en roedor (Caviness, 1975; Wright \& Kern, 1992).

Desde el punto de vista histológico el área 17 de la rata presenta una capa I delgada claramente identificable, conteniendo pocos perfiles celulares. La separación de las capas II y III es poco distinguible y ambas se extienden hasta cerca de un $45 \%$ de la profundidad de la corteza. Bajo ellas se encuentra la capa IV que se caracteriza por ser prominente (de alrededor de $1490 \mu \mathrm{m}$ de espesor) y poseer neuronas pequeñas; alcanza hasta el 55\% de la profundidad de la corteza. La capa V contiene cuerpos celulares de tamaño medio y grande. La capa VI se divide en dos subcapas, la capa VIa, con neuronas pequeñas, de aspecto muy similar a la capa IV, con las células dispuestas en líneas verticales; y, la capa VIb de situación más profunda, que también contiene células pequeñas, pero en este caso su disposición es horizontal (Peters \& Kara, 1985a; 1985b).

La densidad neuronal fue cuantificada, mediante la utilización de la técnica del disector óptico (Braendgaard $e t$ al., 1990). El conteo se realizó en las capas IV y V y se contabilizaron principalmente neuronas piramidales. Las neuronas corticales se identifican principalmente, por su perfil y la presencia de un pequeño nucléolo. Se descartaron las células gliales, la que se distinguen por presentar un perfil nuclear pequeño, de forma irregular e intensamente teñido, así como también las células endoteliales, las que tienen forma oblongada y se encuentran asociadas a capilares (Sterio, 1984).

El conteo se realizó con el aumento mayor del microscopio (100x), en un volumen tisular de $64.000 \mu \mathrm{m}^{3}$. La densidad se expresó finalmente en neuronas $/ \mathrm{mm}^{3}$.

El procedimiento se realizó siempre en el mismo hemisferio cerebral y aproximadamente en el mismo punto, a fin de evitar posibles efectos de lateralización que pudiesen sesgar los resultados, ya que se ha visto, por ejemplo, que la malnutrición proteica tiene un mayor efecto sobre el desarrollo de la corteza occipital lateral que sobre la corteza dorsal de la misma región (Soto-Moyano et al.).

Se emplearon un total de 8 encéfalos (Octodon degus $\mathrm{n}=5$; Octodon bridgesi $\mathrm{n}=3$ ). En cada encéfalo se trabajó con un promedio de 12 cortes y en cada uno de ellos se efectuaron 4 mediciones distintas, las que se promediaron para obtener un valor de densidad por corte.

Complementariamente, se realizó también una 
cuantificación de la densidad neuronal del área dorso medial de la corteza motora (capas III y V) de las especies en estudio (Caviness; Wright \& Kern). Los valores de densidad neuronal se registraron y caracterizaron para su análisis estadístico, para el cual se empleó la prueba de t de Student, de muestras independientes.

\section{RESULTADOS}

La densidad neuronal promedio (neuronas/mm3) de la corteza visual primaria (área 17) se observan en la Tabla I. En la Tabla II se observa la densidad neuronal promedio, de la corteza motora.

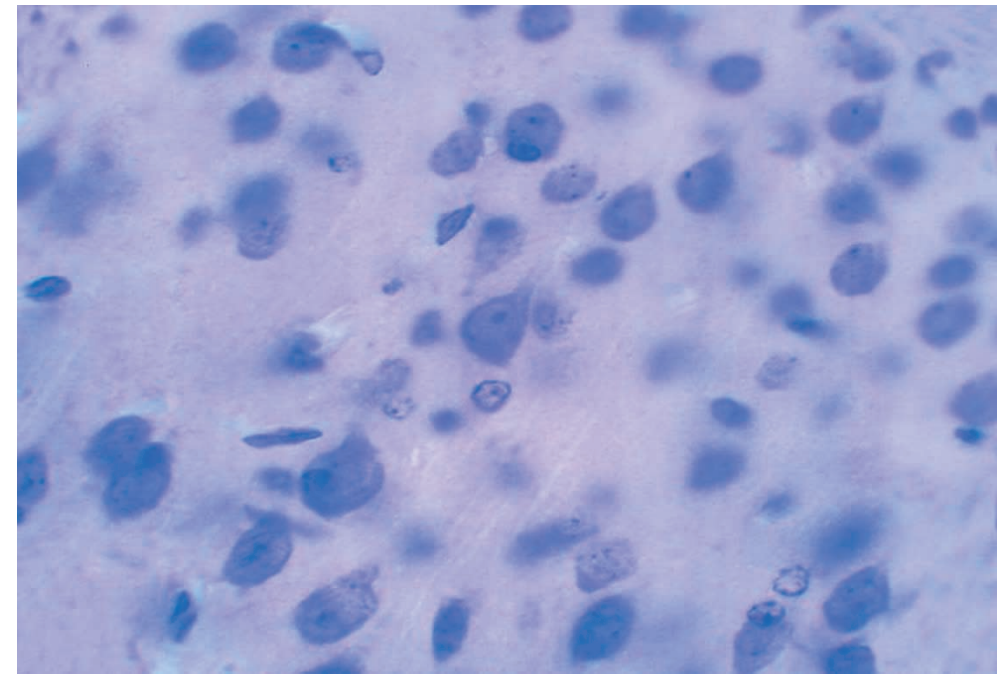

Fig. 2. Neuronas piramidales de la capa V, de la corteza cerebral visual de Octodon bridgesi, Nissl (100X).
Tabla I. Densidad neuronal promedio (neuronas $/ \mathrm{mm}^{3}$ ), de la corteza visual primaria (área 17), de las especies en estudio.

\begin{tabular}{lc}
\hline Especie & Densidad Promedio $\left(\right.$ neuron as $\left./ \mathbf{m m}^{3}\right)$ \\
\hline Octodon degus $(\mathrm{n}=5)$ & $34,32 \pm 2,51 \times 10^{4}$ \\
Octodon bridgesi $(\mathrm{n}=3)$ & $39,55 \pm 0,64 \times 10^{4}$ \\
\hline
\end{tabular}

$\mathrm{p}<0,05 \quad \mathrm{t}=3,44 \quad(\mathrm{p}=0,0138)$

Tabla II. Densidad neuronal promedio (neuronas/mm3), de la corteza motora de las especies en estudio

\begin{tabular}{lc}
\hline Especie & Densidad Promedio (neuronas $/ \mathbf{m m}^{\mathbf{3}}$ ) \\
\hline Octodon degus $(\mathrm{n}=5)$ & $18,67 \pm 2,42 \times 10^{4}$ \\
Octodonbridgesi $(\mathrm{n}=3)$ & $23,70 \pm 1,40 \times 10^{4}$ \\
\hline
\end{tabular}

$\mathrm{p}<0,05 \mathrm{t}=3,21 \quad(\mathrm{p}=0,0184)$

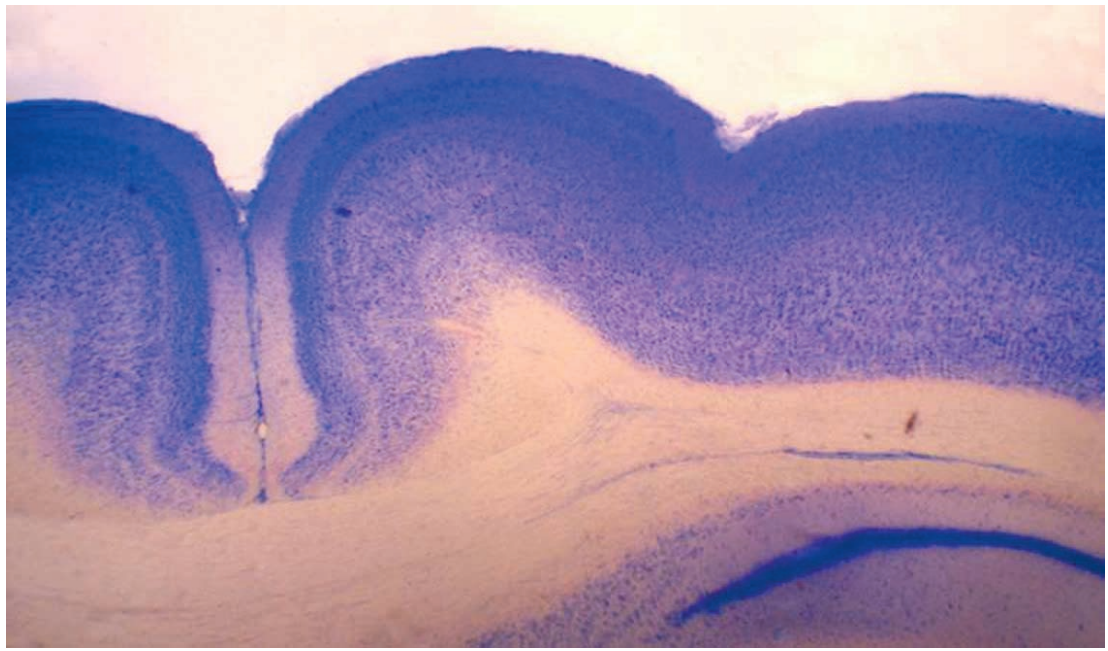

Fig. 1. Corteza cerebral visual de Octodon degus, Nissl (4X).
Característica morfológicas de la corteza cerebral visual del Octodon degus (Fig. 1) y neuronas piramidales de la capa V, de la corteza cerebral visual de Octodon bridgesi (Fig. 2).

\section{DISCUSIÓN}

Nuestros resultados indican que $O$. degus posee una menor densidad neuronal que $O$. bridgesi tanto en la corteza visual como en la motora (Tablas I y II). Por otro lado, O. degus posee también un cerebro mas pequeño $(2,7 \pm 0,2 \mathrm{~g})$, y por ende un coeficiente de encefalización menor (peso encefálico/peso corporal) que $O$. bridgesi, lo cual contrasta con la conocida relación inversa entre densidad neuronal y tamaño cerebral en las distintas especies de mamíferos (Jerison 1973); sin embargo, a pesar de no ser estadísticamente significativa, la diferencia entre los pesos corporales promedios de los O. degus y $O$. bridgesi, hay que considerar el bajo peso de los ejemplares de esta última especie, capturados a terreno $(94,3 \pm 9,2 \mathrm{~g})$, en relación a lo reportado por otros autores (OcampoGarcés et al., 2005). Es decir, las diferencias en densidad neuronal entre ambas especies no se explican por las diferencias en tamaño cerebral o encefalización. En este sentido, es posible que las diferencias obtenidas sean producto de la diversidad en modos de 
vida de ambas especies de roedores silvestres. $O$. degus es una especie diurna y cavadora, lo que podría explicar la disminución de la densidad neuronal en las áreas visual y motora, respectivamente, ya que se ha demostrado una disminución de la densidad neuronal asociada al aumento de la función motora o sensorial.

Es así como, Fernández et al. (2003), encontraron una expansión territorial del campo dendrítico en neuronas observadas en la región lateral de la corteza parieto-occipital, de ratas sometidas a un enriquecimiento polisensorial temprano. Del mismo modo, Morales et al. (1999), demostraron que las ratas que aprendían una habilidad motora, presentaban una disminución permanente en la densidad neuronal de la corteza motora. Sin embargo, para confirmar dicha posibilidad se hará necesario hacer un análisis de una tercera área cortical, cuyas funciones no muestren una especialización sensorial o conductual como las que ya hemos señalado.

Por lo tanto, es posible que la mayor densidad neuronal que se observó en Octodon bridgesi se deba a un menor tamaño de los somas neuronianos, acompañado de una disminución en el número de conexiones y ramificaciones dendríticas. Estudios previos han demostrado que estos parámetros tienen una relación inversa (densidad neuronal versus tamaño de somas y de conexiones dendríticas) (SotoMoyano et al.).

Olivares et al., al comparar el área 17 en dos especies mas distantes filogenéticamente, de roedores silves- tres chilenos, con distintos períodos de actividad circadiana (Phyllotis darwini, con actividad nocturna, y Abrothrix olivaceus, con actividad continua), observaron una mayor densidad neuronal en $P$. darwini que en A. olivaceus, lo que concuerda con este estudio. Además, los ejemplares de $P$. darwini utilizados en dicho estudio presentaron un mayor tamaño cerebral que A. olivaceus, lo que sugiere que esta mayor densidad no se debe a un volumen cerebral menor, sino que podría deberse a las diferencias en actividad circadiana.

Las condiciones naturales de luz y oscuridad en que se desenvuelven las diferentes especies animales podrían influir en el desarrollo de la corteza cerebral occipital, zona destinada al procesamiento de la información relativa a la visión. Esto se ve avalado por estudios previos que han demostrado la importancia que los cambios ambientales y nutricionales durante la gestación y el período postnatal temprano pueden tener sobre el desarrollo definitivo de la corteza cerebral (Fernández et al., 1993).

Los resultados arrojados en este estudio indican los potenciales efectos que podrían tener los períodos de actividad y, por ende, las condiciones de luminosidad en que se desenvuelven las especies animales, en la anatomía de la corteza cerebral, en particular en la densidad neuronal. Sin embargo, serán necesarios estudios adicionales en otras áreas corticales, además de la motora, para poder confirmar esta hipótesis, como también un mayor número de animales analizados.

OLIVARES, R.; ORTÍZ, A.; HENRÍQUEZ, M.; ADARO, L. \& ABOITIZ, F. Neuronal density in primary visual cortex (17 visual area), in two species of octodon. Int. J. Morphol., 28(1):249-253, 2010.

SUMMARY: Studies show that environmental modifications can produce profound alterations in the normal development of the visual cortex and its connectivity. For the other hand it is possible that in natural conditions, animal species have developed genetic adaptations to the different conditions of luminance in which they normally behave. Recently have observed significant changes in cortical neuronal density of area 17 (primary visual area), in two sympatric Chilean rodents with different daily activity (Phyllotis darwini and Abrothrix olivaceus), but have not yet determined the genetic nature or plastic such differences. In this paper we compared species with a closer phylogenetic relation so as to minimize the taxonomic variable. We studied the primary visual cortex (area 17) of wild rodents native of the species Octodon degus $(\mathrm{n}=5)$ and Octodon bridgesi $(\mathrm{n}=3)$, belonging to the Octodontidae family, in order to show changes in the neuronal density, using celloidin-embedded, $40 \mu \mathrm{m}$-thickness Nissl sections, with the aid of an optical dissector. In addition, we performed a quantification of the neuronal density of the motor cortex of the species under study. $O$. degus, bearing a crepuscular-diurnal activity pattern, showed a lower neuronal density in the visual cortex $\left(34.32 \pm 2.51 \times 10^{4}\right.$ neuron $\left./ \mathrm{mm}^{3}\right)$ than that observed in 0. bridgesi $(39.55 \pm$ $0.64 \times 10^{4}$ neuron $\left./ \mathrm{mm}^{3}\right)$, a species that exhibits a nocturnal phase preference, which was statistically significant $(\mathrm{t}=3.44$; $\mathrm{p}<0.05)$. These differences might be related to differences in daily activity in two species, but we cannot discount the influence of other factors.

KEY WORDS: Cerebral cortex; Visual cortex; Neuronal density; Octodon degus; Octodon bridgesi

\section{REFERENCIAS BIBLIOGRÁFICAS}

Argandoña, E. G. \& Lafuente, J. V. Influence of visual experience deprivation on the postnatal development of the microvascular bed in layer IV of the rat visual cortex. Brain Res., 855:137-142, 2000. 
Braendgaard, H.; Evans, S. M.; Howard, C. V. \& Gundersen, H. J. G. The total number of neurons in the human neocortex unbiasedly estimated using optical dissector. J. Microsc., 157:285-304, 1990.

Caviness, V. S. Jr. Architectonic map of neocortex of the normal mouse. J. Comp. Neurol., 164:247-64, 1975.

Felsenstein, J. Phylogenesis and comparative method. Am. Nat., 124:1-15, 1985.

Fernández, V.; Fernández, B. \& Adaro, L. Early polisensorial enrichment: specific experience induced structural changes in the parieto-occipital cortex of the rat. Growth Dev. Aging, 67:3-10, 2003.

Fernández, V.; Pascual, R. \& Ruiz, S. Early - life environmental deterioration, nutrition and ontogenesis of the motor cortex in the rat: a Golgi study. Biol. Neonate, 64:245-53, 1993.

Hernández, F.; Ocampo-Garcés, A. \& Vivaldi, E. A. REM sleep in Octodon degus under entrained conditions. Biol. Res., 36:R96, 2003.

Honeycutt, R. L.; Rowe, D. L. \& Gallardo, M. H. Molecular systematics of the South American caviomorph rodents: relationships among species and genera in the family Octodontidae. Mol. Phylogenet. Evol., 26:476-89, 2003.

Jerison, H. J. Evolution of the Brain and Intelligence. New York, Academic Press, 1973. pp.296.

Kenagy, G. J.; Nespolo, R. F.; Vásquez, R. A. \& Bozinovic, F. Daily and seasonal limits of time and temperature to activity of degus. Rev. Chil. Hist. Nat., 75:567-81, 2002.

Morales, P.; Pinto-Hamuy, T.; Fernández, V. \& Díaz, E. Persistent neuronal density changes related to the establishment of a motor memory. Behav. Brain. Res., 99: 115-121, 1999.

Ocampo-Garcés, A.; Hernández, F.; Mena, W. \& Palacios, A.G. Wheel-running and rest activity pattern interaction in two octodontids (Octodon degus, Octodon bridgesi). Biol. Res., 38:299-305, 2005.

Olivares, R.; Godoy, G.; Adaro, L. \& Aboitiz, F. Densidad neuronal de la corteza visual primaria (área 17), de dos especies de roedores silvestres. Int. J. Morphol., 22:27984, 2004.
Peters, A. \& Kara, D. The neuronal composition of area 17 of rat visual cortex. I. The pyramidal cells. J. Comp. Neurol., 234:218-41, 1985.

Peters, A. \& Kara, D. The neuronal composition of area 17 of rat visual cortex. II. The nonpyramidal cells. J. Comp. Neurol., 234:241-63, 1985.

Soto-Moyano, R.; Fernández, V.; Sanhueza, M.; Belmar, J.; Kusch, C.; Pérez, H.; Ruiz, S. \& Hernández, A. Effects of mild protein prenatal malnutrition and subsequent postnatal nutritional rehabilitation on noradrenalina release and neuronal density in the rat occipital cortex. Dev. Brain Res., 116:51-8, 1999.

Sterio, D. The unbiased stimation of number and sizes of arbitrary particles using the dissector. J. Microsc., 134:127-36, 1984.

Verzi, D. H. \& Alcover, A. Octodon bridgesi, Waterhouse 1844 (Rodentia: Octodontidae) in the Argentinian living mammalian fauna. Mammalia, 54:61-7, 1990.

Wright, J. W. \& Kern, M. D. Stereotaxic atlas of the brain of Octodon degus. J. Morphol., 214:299-320, 1992.

Yakovlev, B. Whole brain serial histological sections. In: C. G. Tedeschi Neuropathology; Methods and Diagnosis. Boston, Little, Brown and Co., 1970. pp.371-8.

Dirección para correspondencia:

Dr. Ricardo Olivares

Avenida Santa Rosa 11735 La Pintana

Correo Postal Casilla 2 Correo 15

La Granja, Santiago

CHILE

Email: rolivare@uchile.cl

Recibido : 02-12-2009

Aceptado: 14-01-2010 
